
\title{
25 Research Soure \\ In - Vivo Conversion of Astrocytes to Neuroblasts in the Injured Spinal Cord
}

\section{Maryam Lale Ataei}

Tabriz Medical University: Tabriz University of Medical Sciences

Mohammad Karimipour ( $\nabla$ karimipourm@tbzmed.ac.ir)

Tabriz University of Medical Sciences https://orcid.org/0000-0002-6975-4308

Parviz Shahabi

Tabriz Medical University: Tabriz University of Medical Sciences

Hamid Soltani-Zangbar

Tabriz Medical University: Tabriz University of Medical Sciences

Maryam PashaiasI

Tabriz Medical University: Tabriz University of Medical Sciences

\section{Research Article}

Keywords: Spinal cord injury, MSCs, Astrocyte, Neuroblast, Reprogramming

Posted Date: April 26th, 2021

DOl: https://doi.org/10.21203/rs.3.rs-362556/v1

License: (c) (1) This work is licensed under a Creative Commons Attribution 4.0 International License.

Read Full License 


\section{Abstract}

Direct astrocyte reprogramming to neural progenitor cells and promotion of neurogenesis is considered as an alternative approach to replace the lost neurons in the spinal cord injury(SCl). Herein, we used the human amniotic fluid mesenchymal stem cells (hAF-MSCs) and their conditioned medium (CM), to investigating their ability to reprogramming astrocytes to neuroblasts following $\mathrm{SCl}$. 54 adult rats were randomly divided into 9 groups $(\mathrm{n}=6)$, included: Control, $\mathrm{SCl},(\mathrm{SCl}+\mathrm{DMEM}),(\mathrm{SCl}+\mathrm{CM}),(\mathrm{SCl}+\mathrm{MSCs})$, (SCl + Astrocyte), (SCl + Astrocyte + DMEM), (SCl + Astrocyte + $\mathrm{CM})$ and SCl + Astrocyte + MSCs). Following laminectomy and $\mathrm{SCl}$ induction, DMEM, CM, MSCs and Astrocytes were injected. Wester-blot was performed to explore the levels of the Sox2 protein in the MSCs-CM. The immunofluorescence staining against DCX and GFAP was done. Finally, Basso-Beattie-Brenham (BBB) locomotor test was conducted to assess the neurological outcomes. Our results showed that the MSCs through juxtacrine and paracrine mechanisms induced the promotion of the endogenous neuroblasts and the decline of astrocytes. Moreover, in the present research, MSCs and CM could convert the transplanted human astrocytes to neuroblasts in the spinal cord injury. Taken together, our data indicate the MSCs via juxtacrine and paracrine pathways could direct the spinal cord endogenous neural stem cells(NSCs) to the neuroblasts lineage rather than astrocytes as well as induce reprogramming. Ultimately, MSCs could reverse the neurobehavioral deficit in the SCI. The striking output in our study was the capability of the MSCs in the reprogramming of the astrocytes to neuroblasts via juxtacrine and paracrine pathways in the In-vivo condition.

\section{Introduction}

Spinal cord injury (SCl) is damage to the spinal cord and a complex neurological condition that resulted in irreversible neuronal loss, glial scar formation, axonal injury, disruption of the myelin sheath and neural tracts and long-lasting disability and in some cases leads to permanent functional deficits in mammals(Su et al. 2014b; Higuchi et al. 2019; Abolhasanpour et al. 2019). Following SCl, astrocytes proliferate and migrate into the surrounding milieu and generate glial scar and undesirable microenvironment (Wang et al. 2016). The research demonstrated that the astrocytes and NG2 (Preoligodrocytes), as a result of reprogramming could convert to neuroblast and neurons in the spinal cord and brain (Wang et al. 2016). It is shown that the SRY (sex- determining region Y-box 2), also known as Sox2, is a transcription factor that is essential for maintaining self-renewal, or pluripotency, of undifferentiated embryonic stem cells. Sox 2 has a critical role in the maintenance of embryonic and neural stem cells and also it is crucial for directing neural differentiation (Zhang and Cui 2014; Mercurio et al. 2019; Rodriguez-Jimenez et al. 2016). Sox2 can mediated reprogramming of astrocytes to DCX + neuroblasts and in this context, Sox 2 can help to treat SCI by converting glial cells to neurons finally mature neurons (Wang et al. 2016; Rodriguez-Jimenez et al. 2016). Doublecortin (DCX) is a microtubuleassociated protein expressed by neuronal precursor cells and immature neurons in embryonic and adult cortical structures and considered as a marker of developing neural progenitor cells (Karimipour et al. 2019; Nasrolahi et al. 2019). Astrocytes due to nearness to radial glial cells identity, their high quantity, 
and the potential to proliferate in the central nervous system, astrocytes could undertake therapeutic interventional approaches including reprogramming. (Sun et al. 2019). Recent advancements in the field of direct in vivo reprogramming showed the generation of functional neurons from reactive glial cells in the repair of the brain ( $\mathrm{Li}$ and Chen 2016; Guo et al. 2014). The concept of direct reprogramming is a process in which one mature somatic cell transforms into another mature somatic cell without undergoing an intermediate pluripotent state or progenitor cell type(Ghasemi-Kasman et al. 2015). Transplantation stem cells including MSCs and olfactory ensheathing cells (OECs) in SCl could lead to increase of neuroprotective molecules and reprogramming of astrocytes into neuroblasts which could survive and develop into mature neurons (Sabapathy et al. 2017; Sun et al. 2019). In this context, MSCs by secretion of the essential materials for neuroprotection such as growth factors, cytokines, and neurotrophic factors, could induce the replacement of lost neuronal cells, remyelination of axons, angiogenesis, and decline of inflammatory responses(Oh and Jeon 2016; Shahrezaie et al. 2017). MSCs could harvest from different sources including adipose derived tissue, bone marrow, Umbilical Cord Wharton's Jelly, amniotic membrane and amniotic fluid mesenchymal stem cells (hAF-MSCs) and so on (Gabel et al. 2017; Oh and Jeon 2016). In spite of the plethora of studies and experiments regarding the evaluation of the functional efficacy of the MSCs in the SCl, the election of the effective source of the MSCs is one of the challenging issues in the scientific community (Gabel et al. 2017). But recent studies, suggest that (hAF-MSCs) could account into consideration in the $\mathrm{SCl}$ because of the amnion origin from the epiblast layer as a pluripotent and undifferentiated structure in the embryo(De Coppi et al. 2007; Antonucci et al. 2014). These cells routinely gained during amniocentesis which is done in the second trimester and express embryonic and pluripotency markers for instance Oct4, Nanog and Sox2 (Aziz et al. 2019; Gholizadeh-Ghaleh Aziz et al. 2019; Dziadosz et al. 2016; Maraldi et al. 2014; Saito et al. 2012). Amniotic fluid derived-MSCs relative to other sources have high proliferative and regenerative potential as well as they maintain pluripotency features, and remain without alteration after continuous passages (Maraldi et al. 2014; Aziz et al. 2016). MSCs during cell culture can secrete paracrine factors in the form of conditioned medium (CM) (Osugi et al. 2012; Cantinieaux et al. 2013). CM contains metabolites, growth factors, extracellular matrix proteins, cytokines and anti-inflammatory agents which are secreted into the medium by the cultured cells. (Cantinieaux et al. 2013; Pawitan 2014). Sox2 which is available in hAF-MSCs-CM is the essential factor for the transform endogenous spinal astrocytes to neuroblasts (Su et al. 2014b; Zhang and Cui 2014). In this Perspective, we investigated the efficiency of the hAF-MSCs and their $\mathrm{CM}$ in the reprogramming of the astrocytes to neuroblasts through juxtacrine and paracrine mechanisms as well as functional behavior following the SCl.

\section{Materials And Methods}

\section{Ethical issue and study design}

In the current study, 54 adult male Wister rats (weight: 270-300g) were purchased from the animal laboratory and maintained according to the guide line of ethics committee of Tabriz University of Medical Sciences (registered number 95/5-10/7). All animals were housed in a standard condition under a $12 \mathrm{~h}$ light/dark schedule with enough food and water. The rats were randomly divided in to 9 groups (six rats 
per group), these nine groups included:Control, SCl, (SCI + DMEM, IP), (SCI + CM, IP), (SCl + MSCs, Focally), (SCl + Astrocyte), (SCl + Astrocyte+ DMEM, IP), (SCl + Astrocyte+ CM, IP) and SCl + Astrocyte+ MSCs). In all groups, laminectomy was performed at the T9-T10 vertebral level in the dorsal surface of the spinal cord using the Infinite Horizons Impactor with an impact force of 150 (moderate SCI) kdyn (1 dyn=1g $\cdot \mathrm{cm} / \mathrm{s} 2=10-5 \mathrm{~kg} \cdot \mathrm{m} / \mathrm{s} 2=10-5 \mathrm{~N}$ ) by impactor device.

\section{hAF-MSCs isolation, cultivation and characterization}

Briefly, isolation of hAF-MSCs was done undergoing amniocentesis for the routine karyotype screening of about $5 \mathrm{ml}$ of amniotic fluid samples from eight mothers in Al-Zahra hospital (Tabriz, Iran). Prior to the amniocentesis, patients written informed consent for donating amniotic fluid samples voluntarily for this research. Amniotic fluid extraction carried out under supervision the gynecologist with using a $22 \mathrm{G}$ Needle. After sending the samples to the desired laboratory, samples were centrifuged at $450 \mathrm{~g}$ for 10 minutes, next the settled pellet was washed twice by PBS (Gibco; Thermo Fisher Scientific, Darmstadt, Germany). Then, the cells transferred in 6 well plates with AmnioMAX II Complete Medium (Gibco, cat\# 11269) for $1-2$ weeks in condition $37^{\circ} \mathrm{C}$ and $5 \% \mathrm{CO} 2$. In the cultivation period, the medium was changed twice a week, and in the $90 \%$ confluence, the cells trypsinized with trypsin-EDTA [0.25\%] (Gibco) and centrifuged, and finally, cells pellet re-seeded in DMEM-low glucose media with $15 \%$ FBS, $1 \%$ penicillin/streptomycin and $10 \mathrm{ng} / \mathrm{mL}$ bFGF in the optimized condition. The phenotype profile of the hAFMSCs was examined by flow cytometry analysis. For this purpose, the cells at passage 3-5 were trypsinzed and two times washed with PBS and centrifuge at 1500 RPM for $3 \mathrm{~min}$, then, the cell pellets were stained with antibodies including CD 105 (Catalog No. 1p-298-To25 Exbio), CD 73 (Catalog No. 561260 BD Biosciences) antibodies as mesenchymal stem cell markers and CD 45 (Catalog No. 1F-222T025 Exbio), CD 14 (Catalog No. 12-0149-42 eBioscience) as hematopoietic stem cell markers) with dilution $1 / 30$ in the PBS for 20 min on ice.

\section{Preparation of CM}

The MSCs at the 3rd-5rd passage and $90 \%$ confluent, were trypsinized and washed with PBS three times, and re-fed with DMEM-low glucose culture medium in serum-free condition for $48 \mathrm{~h}$. Then, CM was harvested and centrifuged at $450 \mathrm{~g}$ for $10 \mathrm{~min}$ up to eliminate free-floating cells. Finally, CM was sterilized through $0.22 \mu \mathrm{m}$ filters and concentrated by freeze-drying processes and was stored at $-80{ }^{\circ} \mathrm{C}$ until use.

\section{Western blot}

$\mathrm{CM}$ was collected from MSCs culture and was sterilized by $0.22 \mathrm{~mm}$ filters, then CM was concentrated 2 , 4, 8, 16 and 32 folds by freeze dryer device. Sox2 (Sex determining region Y-box 2) secreted by HAF-MSCs into MSCs-CM, was measured using western blot analysis.

\section{Human astrocyte culture}

Human astrocytes (line 1321N1) (Ghasemi-Kasman et al. 2015) were purchased from Pasteur Institute of Iran and cultured in DMEM low glucose medium with $10 \% \mathrm{FBS}$ and $1 \%$ penicillin/streptomycin. This 
medium was changed twice in a week.

\section{Spinal Cord Surgery}

The rats were anesthetized by inhalation of $5 \%$ isoflurane and oxygen $(1 \mathrm{~L} / \mathrm{min})$ in a closed space. After deep anesthesia, an adequate level of anesthesia was determined by checking withdrawal to painful stimuli applied to the hind limb. The rats were shifted to the surgical location and via the mask received an isoflurane vapor inhalation (3-5\%) and oxygen (0.8-1 L/min) to the end of surgical procedure. Briefly, animals under anesthesia conditions, their back was shaved and in the midline, the skin $\square s$ incision was performed around $2 \mathrm{~cm}$, and in order to laminectomy, Paravertebral muscles were cut up on the T9-T11 spinal processes, and with the dental drill, a hole of $1.5 \mathrm{~mm}$ diameter was made in the vertebral arch of T10 as far as dura mater could be seen. Then, using the Horizons Impactor, animals received a force of 150-kilodyne (moderate $\mathrm{SCl}$ ) on the targeted spinal cord segments, subsequently, the muscles and skin were closed. Also for bilateral injury, transverse process of the rats throughout the surgery and injury fixed by a clamp. To prevent infection following SCl, ciprofloxacin (350 ml units/days) was injected via IP for one week. After $\mathrm{SCl}$, the bladder sac was discharged manually twice a day for one week.

\section{Infusion of MSCs- CM}

In order to explore the effect of the $\mathrm{CM}$ on the rate expression of the endogenous neuroblasts and astrocytes, $500 \mu \mathrm{l}$ of $\mathrm{CM}$, following $\mathrm{SCl}$ was infused through intraperitoneal (IP) per day for 7 days.

\section{Transplantation of hAF-MSCs}

The next, to examine the effect of the hAF-MSCs on endogenous expression of neuroblasts and astrocytes, the MSCs were detached and harvested using $0.25 \%$ Trypsin-EDTA. Prior to the transplantation, the number of cells was estimated by counting in a neobar lam and washed by PBS three times. Following SCl, 5×105 cells per $5 \mu \mathrm{l}$ PBS were transplanted to the proximal, central, and distal parts of the lesion site using a capillary glass needle through a Hamilton syringe. For immunosuppression, the rats received cyclosporine ( $1 \mathrm{mg} / 100 \mathrm{~g}$ body weight) two days before cell transplantation until the end of the experiment(Springer et al. 2018).

\section{Injection of the exogenous human astrocytes}

In the next series of experiments, we decided to investigate the effect of the MSCs and their CM on astrocyte reprogramming and converting the human astrocytes to neuroblasts. To this end, the human astrocytes (Cell line 1321N1) were injected focally into the lesion site of the spinal cord concomitantly with transplantation of MSCs and infusion of CM.

\section{Tissue preparation and immunofluorescence staining}

For immunofluorescence examinations, animals were sacrificed two weeks after $\mathrm{SCl}$ by ketamine $(100 \mathrm{mg} / \mathrm{kg})$ and xylazine $(5 \mathrm{mg} / \mathrm{kg})$ overdose. The rats were transcardially perfused with normal saline 
( $\mathrm{NaCl} 9 \%)$ and $4 \%$ paraformaldehyde, respectively. The animal's spinal cord was carefully removed and post-fixed overnight with $4 \%$ paraformaldehyde solution. Then, samples of the spinal cord were processed and embedded in paraffin and $5-\mu \mathrm{m}$ thick histological sections prepared by microtome and mounted on poly-lysine-coated slides. After overnight incubation at $4{ }^{\circ} \mathrm{C}$ temperature, the sections were deparaffinized and rehydrated in decreasing ethanol and washed in tap water, and finally stored at $4 \circ \mathrm{C}$ until use. Following the washing in PBS $(0.1 \mathrm{M}, \mathrm{pH} 7.4$, and $0.9 \% \mathrm{NaCl})$, antigen retrieval was done by incubating the sections in preheated $10 \mathrm{mM}$ sodium citrate buffer for $15 \mathrm{~min}$ at $100{ }^{\circ} \mathrm{C}$ and blocking endogenous peroxidase step was performed using incubation the sections in $0.6 \% \mathrm{H} 2 \mathrm{O} 2$ in PBS for 30 min. Then the Sections were exposed to the primary antibodies, Anti- Doublecortin antibody (ab18723), Anti-GFAP antibody [2A5] (ab 4648), Human Anti- Doublecortin antibody (A83146) and Human GFAP antibody MAB2594, for overnight at $4{ }^{\circ} \mathrm{C}$. After three times PBS wash, the sections were incubated with secondary antibodies including Goat Anti-Mouse IgG H\&L (Phycoerythrin) (ab 97024), Goat Anti-Rabbit IgG H\&L (FITC) (ab 6717), Donkey Anti-Goat IgG H\&L (FITC) (ab6881) and Goat Anti-Mouse IgG H\&L (Texas Red $®$ ) (ab6787) at room temperature for 1 hour. Also, nuclei were counterstained with 4', 6diamidino-2-phenylindole (DAPI) (ab 104139). Images were taken and observed by an Olympus fluorescence microscope and data was analyzed using the Image $\mathrm{J}$ program software plugin.

\section{Neurobehavioral examination}

Locomotor performance on 1, 7 and 21 days after $\mathrm{SCl}$ was assessed with the use of the 21 point (a score from 0 (complete paralysis) to 21 (normal gait)) BBB (Basso, Beattie and Bresnahan) score by two examiners blinded.

\section{Statistical analysis}

The results were presented as mean \pm SEM. One-way analysis of variance (ANOVA) and post hoc Tukey tests were performed to detect the statistically significant difference between groups. $P<0.05$ was considered as statistically significant. All the statistics presented in the article were analyzed and drawn using Graph Pad Prism (version 6.01; Graph Pad Software, CA, USA).

\section{Results}

\section{Flow cytometry analysis and phenotype acquisition}

In this research, isolated and cultivated of MSCs from human amniotic fluid showed spindle-like morphology and fibroblast-like under bright-field microscope. Evaluation of cell surface markers of the MSCs at Passages (3-5) by flow cytometry analysis revealed that the MSCs express CD105 (95.80\%) and CD73 (92.2\%) as the MSCs markers while they didn't express, CD14 (9.90\%) and CD $45(9.16 \%)$ as the hematopoietic markers (Figure 1).

\section{MSCs secreted the SOX2 protein in the CM}


To evaluate of the Sox 2 protein existence as a pluripotency marker in the CM by MSCs, Western blot experiment was performed. This analysis showed that the Sox 2 protein was observed in the 32 folds concentrated CM. This finding indicated that the MSCs release the Sox 2 protein in their condition media. (Figure 2).

\section{hAF-MSCs increased endogenous neuroblasts and decreased astrocytes via juxtacrine and paracrine mechanisms.}

For this purpose, immunofluorescence staining was conducted (Figure 3. A). The results from this panel showed that the MSCs after two weeks of transplantation promoted the level of DCX expression and suppressed the rate of GFAP marker. Data analysis showed significant differences among the groups (Figure 3.A, B). Our experiment revealed that the $\mathrm{SCl}$ significantly decreased the number of neuroblasts relative to the control group, but and increased the quantification of astrocytes compared to the control group ( $p<.001$ ) (Figure 3.A, B). MSCs transplantation significantly increased the number of neuroblasts relative to the $\mathrm{SCl}$ and $\mathrm{SCl}+\mathrm{DMEM}$ groups $(\mathrm{p}<.001)$ meanwhile the expression level of astrocytes at the protein level in the MSCs group was significantly decline relative to the $\mathrm{SCl}$ and $\mathrm{SCl}+\mathrm{DMEM}$ groups $(p<.001)$ (Figure 3.A, B). In our study, the increase of the number of neuroblasts and decline astrocytes under influence of MSCs was significant relative to the CM $(p<.05)$. Moreover, $\mathrm{CM}$ could increase the number of neuroblasts and decreased the number of astrocytes compared to the DMEM group $(p<.01)$ (Figure 3.A, B). In general, these data indicate the MSCs through juxtacrine and paracrine pathways could promote the neuroblasts and induce neurogenesis, as well as, diminish the astrocytes in the $\mathrm{SCl}$ and finally suppress gliosis and glial scar formation. Ultimately, it is concluded that the MSCS could direct the fate of endogenous neural stem cells into neural lineage and neurogenesis by involving the juxtacrine and paracrine pathways in the SCl.

\section{hAF-MSCs converted the injected astrocytes to neuroblasts through juxtacrine and paracrine pathways in the in-vivo condition.}

Our main aim from this panel was whether the MSCs and their CM could convert the transplanted human astrocytes to neuroblasts in the in-vivo? To this end, immunofluorescence staining against GFAP and DCX markers was performed (Figure 4. A). The results showed that there was a significant difference among groups after two weeks of cell transplantation (Figure 4.A, B). The number of neuroblasts was significantly increased in the ( $\mathrm{SCl}+$ Astrocytes+ MSCs, $\mathrm{p}<.001)$ and $(\mathrm{SCl}+$ Astrocytes and $\mathrm{CM}, \mathrm{p}<.05)$ versus the ( $\mathrm{SCl}+$ Astrocytes) and ( $\mathrm{SCl}+$ Astrocytes + DMEM) (Figure 4.A, B). In this study, MSCs and CM could increase the number of neuroblasts relative to the (SCI +Astrocytes + DMEM) group $(p<.001, p<.05)$ respectively (Figure 4.A, B). In this context, the raise of neuroblasts in the (SCl +Astrocytes+ MSCs) group was significantly more than (SCl+ Astrocytes and $\mathrm{CM})(\mathrm{p}<.01)$ (Figure 4.A, B).

The other aim in the current research was the evaluation of the effect of the MSCs and CM on the number of the transplanted human astrocytes in the in-vivo condition. Our data showed that the MSCs and CM significantly reduced the number of the transplanted human astrocytes in the ( $\mathrm{SCl}+$ Astrocytes+ MSCs $\mathrm{p}<.001)$ and $(\mathrm{SCl}+$ Astrocytes $+\mathrm{CM}, \mathrm{p}<.05)$ groups relative to the (SCl+ Astrocytes) group as well as the 
$(\mathrm{SCl}+$ Astrocytes+ MSCs $\mathrm{p}<.001)$ and $(\mathrm{SCl}+$ Astrocytes $+\mathrm{CM}, \mathrm{p}<.05)$ groups compared to the $(\mathrm{SCl}+$ Astrocytes+ DMEM) group (Figure 4.A, B). It should be considered that the number of astrocytes in the ( $\mathrm{SCl}+$ Astrocytes+ MSCs) group was significantly less than those in the (SCl+ Astrocytes + $\mathrm{CM}$ ) group $(p<.05)$ (Figure 4.A, B). Taken together, these striking findings indicate that the MSCs through secretion of the important and essential molecules for example SOX 2 and other materials by the mediation of juxtacrine and paracrine pathways could convert the astrocytes to neuroblasts and overall contributed to neuroblasts maturation, neurogenesis, and ultimately neural neuroregeneration in the SCl.

\section{hAF-MSCs promoted the behavioral performance by the mediating of juxtacrine and paracrine mechanisms in the SCl injury.}

In the present study, $\mathrm{SCl}$ was resulted in complete hindlimb motor function paralysis, as shown in (Figure 5). The BBB score as an indicator of functional performance slowly increased between 1 and 5 score at 1 week in all groups. In this section, we evaluated the functional recovery in two panels based on our groups (Figure 5.A, B). In Panel A (Figure 5.A), we would like to assess the effects of the transplantation of MSCs and CM on the score of the BBB test and functionality and in panel B (Figure 5.B), the functional efficiency of the reprogrammed neuroblasts from the grafted exogenous astrocytes was examined.

In panel A, MSCs and CM could promote functional recovery after two weeks' cell and CM infusion. Regarding this, our data showed that the MSCs significantly increased the score of the functional behavior relative to the ( $\mathrm{SCl}$ and, $\mathrm{SCl}+\mathrm{DMEM},(\mathrm{p}<.001))$ groups as well as compared to the (SCl+ $\mathrm{CM})$ group $(p<.05)$ (Figure 5.A). In the present research, $\mathrm{CM}$ could increase the functional recovery in the $(\mathrm{SCl}+$ CM group) relative to the (SCl+ DMEM) group $(p<.01)$ (Figure 5.A).

In panel $\mathrm{B}$, the results from the BBB test evaluation indicated that the score of the functional recovery under influence of reprogrammed neuroblasts significantly increased in the (SCl+ Astrocytes + MSCs) group relative to the (SCl+ Astrocytes) and (SCl+ Astrocytes+ DMEM) groups( $\mathrm{p}<.001)$ (Figure 5.B). Also, in our experiments, the functional recovery in the ( $\mathrm{SCl}+$ Astrocytes $+\mathrm{CM})$ group under influence of the reprogrammed neuroblasts significantly promoted relative to the (SCl+ Astrocytes + DMEM) group $(p<.05)$ (Figure 5.B). Moreover, presented data showed that MSCs in comparison to CM could raise the functional recovery score more than the $\mathrm{CM}(\mathrm{p}<.05)$ (Figure 5.B). Altogether, MSCs by the mediating of juxtacrine and paracrine mechanisms contributed to increasing the endogenous neuroblasts and exogenous astrocytes reprogramming to neuroblasts and finally could rescue the functional deficit in the $\mathrm{SCl}$.

\section{Discussion}

Astrocytes in large quantities have been distributed throughout the CNS and provide essential factors and desire microenvironment for optimal neural tissue structure and function in a healthy condition(Sofroniew and Vinters 2010). In response to any CNS damage, they are activated, proliferated, migrated to the lesion site, and participate in glial scar formation (Sofroniew 2009; Yiu and He 2006; Buffo et al. 2008). In the early phase of neuronal injury, these reactive astrocytes exert the lesion sealing effects, which followed by creating a mechanical and biochemical obstacle in a later stage to axonal 
regeneration (Okada et al. 2006). A plethora of studies has shown that the activated astrocytes in CNS injuries could be isolated and cultivated in vitro cultivation and produce neurospheres with the capability of a generation of neurons, astrocytes, and oligodendrocytes. (Buffo et al. 2008; Nakagomi et al. 2009; Su et al. 2014b; Ghasemi-Kasman et al. 2015). Regardless of these findings, astrocyte-derived neurons have not identified around the injured area in both the brain and spinal cord (Ohori et al. 2006; Buffo et al. 2008; Buffo et al. 2005). During gliosis, is observed an elevation in the levels of GFAP and cyclooxygenase (COX)-2 in the astrocytes, as well as other factors like nitric oxide (NO), IL-6, and TNF-a increased in astrocytes and microglia. (Langley et al. 2005). A similar study demonstrated that following $\mathrm{SCl}$ or demyelination in the spinal cord, oligodendrocyte and ependymal cells can increase Sox 2 expression, which is followed by Sox 2 binding to regulatory regions of several genes involved in regulating reactive astrocyte action (Chen et al. 2019). The previous studies have demonstrated that the neural stem and progenitor cells after grafting into the undamaged spinal cord migrate in a rostral or caudal direction and two months later, a significant proportion of these transplanted cells differentiated into GFAP-positive astrocytes, and just a low percentage remained as undifferentiated NSCs (Falnikar et al. 2015). Therefore, finding new and effective therapeutic strategies including reprogramming and neurogenesis promotion is a challenging and debate subject in the regenerative medicine field. However, focusing on these data, it could be clear that the best targets for reprogramming of in vivo linage might be endogenous astrocytes, and in vivo reprogramming non-neuronal cells mainly astrocytes to neuroblasts has been considered at the center of attention in the scientific community (Guo et al. 2014; Torper et al. 2013; Niu et al. 2013; Grande et al. 2013). Previous studies have indicated the feasibility and efficiency of the MSC-derived CM in the models of acute brain injuries and also highlighted that the route of administration could have an impact on the degree of protection (Deng-Bryant et al. 2012; Deng-Bryant et al. 2015). As the enriched source of MSCs/CM, hAF-MSCs hold several advantages due to high cell recovery, noninvasive preparation and pluripotency characteristics (Fierabracci et al. 2015). Pischiutta et al. to investigating the effects of Ah-MSCs-Secreted Metabolites on brain injuries have shown that hAMSC and CM treatments exert protective effects on the cortical region of acute brain injury (Pischiutta et al. 2016). In the present study, we explored the juxtacrine and paracrine effects of the hAF-MSCs in the reprogramming of the endogenous and exogenous astrocytes into neuroblasts in adult male rats after $\mathrm{SCl}$. According to previous evidence, transcription factor Sox 2 could be sufficient to converting endogenous differentiated astrocytes into neuroblasts and also mature neurons in the adult spinal cord with different severity of the injury (Su et al. 2014a). Currently, it is well documented that the Sox2 is a single transcription factor that leads to the reprogrammed of astrocytes into active neurons (GhasemiKasman et al. 2015). Also, the factor of Sox2, has a critical role in brain development, neurogenesis, and synapse-forming interneurons(Su et al. 2014b). Therefore, in the current research, Sox 2 was considered as a key factor in astrocyte reprogramming to neuroblasts. Since in the adult spinal cord there are a few newly produced neurons, researchers believe that adult spinal cord-dwelling astrocytes could generate neuroblasts and mature neurons using reprogramming methods(Su et al. 2014b; Li and Chen 2016). Several previous studies have used CM in various disorders like skin wounds (Chen et al. 2008), CNS (Teixeira et al. 2014), hepatic transplant (Du et al. 2013), acute lung injury (lonescu et al. 2012), and chronic kidney diseases (van Koppen et al. 2012). The expression of the cell surface markers of the 
isolated and cultivated MSCs in the present study was consistent with the previous researches (Markmee et al. 2017; Dominici et al. 2006), So that, the MSCs at 3-4 passage, were positive for MSCs including CD105 (95.80\%) and CD73 (92.2\%) markers meanwhile they were negative for CD14 (9.90\%) and CD45 (9.16\%) markers for hematopoietic stem cells. Western-blot analysis showed that the harvested CM from the hAF-MSCs expresses the SOX 2 protein as a main driver in astrocyte reprogramming to DCX-positive neuroblasts. DCX is a microtubule-associated protein expressed predominantly in neuroblasts, immature neurons in developmental processes, and most importantly, in the neurogenic area of the adult brain (Brown et al. 2003; Gleeson et al. 1999). Expression of DCX has a relationship with neurogenesis but didn't observed in regenerative axonal growth or reactive gliosis (Couillard-Despres et al. 2005). Consistent with these findings, in the current study, DCX was very low in spinal cord induced injuries. Our data revealed that the MSCs and CM could significantly increase the number of endogenous neuroblasts and reduced the number of endogenous astrocytes in the injured spinal cord. Although in this context, the effect of the MSCs was more in comparison to CM. It is noteworthy that the MSCs release the critical factors in the $\mathrm{CM}$ and thereby could play a major role in the astrocyte reprogramming to neuroblasts. In line with our research, Su et al have shown that the SOX 2 exclusively could induce the conversion of astrocytes to neuroblasts and MAP2- positive mature neurons in the adult spinal cord. (Su et al. 2014a). In cell therapy, stem cells are transplanted into the tissue to exert effects on treating a disease with or without gene therapy (Abolhasanpour et al. 2020). Regarding this issue, the MSCs have commonly used in the treatment of various diseases in the clinic, and prominently in animal studies were used in the treatment of tissue injury as well as immune disorders. (Salehi-Pourmehr et al. 2019). In-vitro studies have shown that the AF-MSCs can differentiate into cells that express neural lineage marker (Tsai et al. 2006). However, following the transplantation into the ischemic rat brain, it was observed that they have the ability to survive, migrate and differentiate into astrocytes and also immature neurons (Cipriani et al. 2007). Supporting these findings, our results showed that after MSCs transplantation in spinal cord injured rats, the level of GFAP decreased, and the level of DCX significantly increased in the MSCs treated group. In the next series of experiments, we decided to explore whether hA-MSCs and CM could covert the exogenous astrocytes to neuroblasts in the in-vivo condition?

To address this key question, we transplanted the human astrocytes concomitantly with MSCs and CM into the rat injured spinal cord and evaluated the fate of the grafted human astrocytes using the immunofluorescence staining technique in the in-vivo. Regarding our results, the majority of the grafted human astrocytes converted to neuroblasts in those groups who have received the MSCs and CM. It should be considered that this converting rate was significantly more in the ( $\mathrm{SCl}+$ Astrocytes + MSCs) group relative to the $(\mathrm{SCl}+$ Astrocytes $+\mathrm{CM})$ group. In-vivo tracing showed that the grafted human astrocytes were significantly was decreased in the (SCl+ Astrocytes + MSCs) group and ( $\mathrm{SCl}+$ Astrocytes $+\mathrm{CM}$ ) groups. But this decline in the number of the transplanted human astrocytes was significantly more in the ( $\mathrm{SCl}+$ Astrocytes + MSCs) group in comparison to the $(\mathrm{SCl}+$ Astrocytes + $\mathrm{CM})$ group. This is indicating that the MSCs compared to CM could better direct the astrocytes reprogramming to neuroblasts and neurogenesis provocation. Overall, this experiment revealed that the MSCs and CM could increase the DCX - positive cells and diminish the GFAP-positive cells in the in-vivo condition and MSCs 
and $\mathrm{CM}$, could be considered as future relevant tools in the field of regenerative medicine (Dominici et al. 2006). Neuhuber et al. [107] showed that CM of BM-MSCs, because of the presence of IL-6, BDNF, SDF1a, VEGF, MCP-1, and SCF, can induced axon growth and functional recovery in SCl (Neuhuber et al. 2005). A plethora of studies shows that away from cell-cell interaction, the immunomodulation and regenerative capacity of MSCs in the lesion site could be the result of the secretome efficacy (Shi et al. 2010). Therefore, these protective and regenerative actions induced by MSCs secreted molecules may explain significant therapeutic effects in all of the CNS(Uccelli et al. 2011).

In conclusion, the results of the present research point out the protective and regenerative potential of MSCs in the SCl through reprogramming astrocytes to neuroblasts by mediating juxtacrine activity and paracrine effects. Astrocytes reprogramming to neuroblasts is a fundamental and intricate phenomenon in the field of regenerative medicine. Our study is preliminary research in this context and up to now cellular and molecular mechanisms underlying this cellular reprogramming are not fully understood and regarding this, more and complementary studies should be done to address all aspects of the reprogramming subject.

\section{Declarations}

\section{Author Contributions}

M.PA and P.SH designed and supervised the whole study. M.K prepared and wrote the manuscript and analyzed data. M.A performed the experiments and involved in data acquisition. H.SZ was involved in animal surgery.

\section{Funding:}

This work was supported by a grant (No: 95/5-10/7) from Tabriz University of Medical Sciences.

\section{Data Availability:}

Data will be available in request.

\section{Compliance with Ethical Standards}

Conflict of interest: The authors declare that they have no competing interests.

\section{Ethical Approval}

All experiments were conducted according to international principal guidelines and approved by a local ethics committee of Tabriz University of Medical Sciences.

Informed Consent 
All of the patients who participated in the current research, prior to sampling donation received the information and all of the considerations regarding the project and filled the informed consent.

\section{References}

1. Abolhasanpour N, Eidi A, Hajebrahimi S, Reyhani-Rad S, Hashim H (2019) Effect of cerebrolysin on bladder function after spinal cord injury in female Wistar rats. International Journal of Urology 26 (9):917-923

2. Abolhasanpour N, Hajebrahimi S, Ebrahimi-Kalan A, Mehdipour A, Salehi-Pourmehr H (2020) Urodynamic Parameters in Spinal Cord Injury-Induced Neurogenic Bladder Rats after Stem Cell Transplantation: A Narrative Review. Iranian Journal of Medical Sciences 45 (1):2

3. Antonucci I, Di Pietro R, Alfonsi M, Centurione MA, Centurione L, Sancilio S, Pelagatti F, D'amico MA, Di Baldassarre A, Piattelli A (2014) Human second trimester amniotic fluid cells are able to create embryoid body-like structures in vitro and to show typical expression profiles of embryonic and primordial germ cells. Cell transplantation 23 (12):1501-1515

4. Aziz SG-G, Fardyazar Z, Pashaei-Asl F, Rahmati-Yamchi M, Khodadadi K, Pashaiasl M (2019) Human amniotic fluid stem cells (hAFSCs) expressing p21 and cyclin D1 genes retain excellent viability after freezing with (dimethyl sulfoxide) DMSO. Bosnian Journal of Basic Medical Sciences 19 (1):43

5. Aziz SG-G, Pashaei-Asl F, Fardyazar Z, PashaiasI M (2016) Isolation, characterization, cryopreservation of human amniotic stem cells and differentiation to osteogenic and adipogenic cells. PloS one 11 (7)

6. Brown JP, Couillard-Després S, Cooper-Kuhn CM, Winkler J, Aigner L, Kuhn HG (2003) Transient expression of doublecortin during adult neurogenesis. Journal of Comparative Neurology 467 (1):110

7. Buffo A, Rite I, Tripathi P, Lepier A, Colak D, Horn A-P, Mori T, Götz M (2008) Origin and progeny of reactive gliosis: A source of multipotent cells in the injured brain. Proceedings of the National Academy of Sciences 105 (9):3581-3586

8. Buffo A, Vosko MR, Ertürk D, Hamann GF, Jucker M, Rowitch D, Götz M (2005) Expression pattern of the transcription factor Olig2 in response to brain injuries: implications for neuronal repair.

Proceedings of the National Academy of Sciences 102 (50):18183-18188

9. Cantinieaux D, Quertainmont R, Blacher S, Rossi L, Wanet T, Noël A, Brook G, Schoenen J, Franzen R (2013) Conditioned medium from bone marrow-derived mesenchymal stem cells improves recovery after spinal cord injury in rats: an original strategy to avoid cell transplantation. PloS one 8 (8):e69515

10. Chen C, Zhong X, Smith DK, Tai W, Yang J, Zou Y, Wang L-L, Sun J, Qin S, Zhang C-L (2019) Astrocyte-specific deletion of Sox 2 promotes functional recovery after traumatic brain injury. Cerebral Cortex 29 (1):54-69 
11. Chen L, Tredget EE, Wu PY, Wu Y (2008) Paracrine factors of mesenchymal stem cells recruit macrophages and endothelial lineage cells and enhance wound healing. PloS one 3 (4):e1886

12. Cipriani S, Bonini D, Marchina E, Balgkouranidou I, Caimi L, Zucconi GG, Barlati S (2007) Mesenchymal cells from human amniotic fluid survive and migrate after transplantation into adult rat brain. Cell Biology International 31 (8):845-850

13. Couillard-Despres S, Winner B, Schaubeck S, Aigner R, Vroemen M, Weidner N, Bogdahn U, Winkler J, Kuhn HG, Aigner $L$ (2005) Doublecortin expression levels in adult brain reflect neurogenesis. European Journal of Neuroscience 21 (1):1-14

14. De Coppi P, Bartsch G, Siddiqui MM, Xu T, Santos CC, Perin L, Mostoslavsky G, Serre AC, Snyder EY, Yoo JJ (2007) Isolation of amniotic stem cell lines with potential for therapy. Nature biotechnology 25 (1):100-106

15. Deng-Bryant Y, Chen Z, van der Merwe C, Liao Z, Dave JR, Rupp R, Shear DA, Tortella FC (2012) Longterm administration of amnion-derived cellular cytokine suspension promotes functional recovery in a model of penetrating ballistic-like brain injury. Journal of Trauma and Acute Care Surgery 73 (2):S156-S164

16. Deng-Bryant Y, Readnower RD, Leung LY, Cunningham TL, Shear DA, Tortella FC (2015) Treatment with amnion-derived cellular cytokine solution (ACCS) induces persistent motor improvement and ameliorates neuroinflammation in a rat model of penetrating ballistic-like brain injury. Restorative neurology and neuroscience 33 (2):189-203

17. Dominici M, Le Blanc K, Mueller I, Slaper-Cortenbach I, Marini F, Krause D, Deans R, Keating A, Prockop D, Horwitz E (2006) Minimal criteria for defining multipotent mesenchymal stromal cells. The International Society for Cellular Therapy position statement. Cytotherapy 8 (4):315-317

18. Du Z, Wei C, Cheng K, Han B, Yan J, Zhang M, Peng C, Liu Y (2013) Mesenchymal stem cellconditioned medium reduces liver injury and enhances regeneration in reduced-size rat liver transplantation. Journal of Surgical Research 183 (2):907-915

19. Dziadosz M, Basch RS, Young BK (2016) Human amniotic fluid: a source of stem cells for possible therapeutic use. American journal of obstetrics and gynecology 214 (3):321-327

20. Falnikar A, Li K, Lepore AC (2015) Therapeutically targeting astrocytes with stem and progenitor cell transplantation following traumatic spinal cord injury. Brain research 1619:91-103

21. Fierabracci A, Lazzari L, Muraca M, Parolini O (2015) How far are we from the clinical use of placental-derived mesenchymal stem cells? Taylor \& Francis,

22. Gabel BC, Curtis El, Marsala M, Ciacci JD (2017) A review of stem cell therapy for spinal cord injury: large animal models and the frontier in humans. World neurosurgery 98:438-443

23. Ghasemi-Kasman M, Hajikaram M, Baharvand H, Javan M (2015) MicroRNA-mediated in vitro and in vivo direct conversion of astrocytes to neuroblasts. PloS one 10 (6)

24. Gholizadeh-Ghaleh Aziz S, Fardyazar Z, Pashaiasl M (2019) The human amniotic fluid mesenchymal stem cells therapy on, SKOV3, ovarian cancer cell line. Molecular Genetics \& Genomic Medicine 7 (7):e00726 
25. Gleeson JG, Lin PT, Flanagan LA, Walsh CA (1999) Doublecortin is a microtubule-associated protein and is expressed widely by migrating neurons. Neuron 23 (2):257-271

26. Grande A, Sumiyoshi K, López-Juárez A, Howard J, Sakthivel B, Aronow B, Campbell K, Nakafuku M (2013) Environmental impact on direct neuronal reprogramming in vivo in the adult brain. Nature communications 4 (1):1-12

27. Guo Z, Zhang L, Wu Z, Chen Y, Wang F, Chen G (2014) In vivo direct reprogramming of reactive glial cells into functional neurons after brain injury and in an Alzheimer's disease model. Cell stem cell 14 (2):188-202

28. Higuchi A, Kumar SS, Benelli G, Ling Q-D, Li H-F, Alarfaj AA, Munusamy MA, Sung T-C, Chang Y, Murugan K (2019) Biomaterials used in stem cell therapy for spinal cord injury. Progress in Materials Science

29. Ionescu L, Byrne RN, van Haaften T, Vadivel A, Alphonse RS, Rey-Parra GJ, Weissmann G, Hall A, Eaton $F$, Thébaud B (2012) Stem cell conditioned medium improves acute lung injury in mice: in vivo evidence for stem cell paracrine action. American Journal of Physiology-Lung Cellular and Molecular Physiology 303 (11):L967-L977

30. Karimipour M, Rahbarghazi R, Tayefi H, Shimia M, Ghanadian M, Mahmoudi J, Bagheri HS (2019) Quercetin promotes learning and memory performance concomitantly with neural stem/progenitor cell proliferation and neurogenesis in the adult rat dentate gyrus. International Journal of Developmental Neuroscience 74:18-26

31. Langley B, Gensert JM, Beal MF, Ratan RR (2005) Remodeling chromatin and stress resistance in the central nervous system: histone deacetylase inhibitors as novel and broadly effective neuroprotective agents. Current Drug Targets-CNS \& Neurological Disorders 4 (1):41-50

32. Li H, Chen $\mathrm{G}$ (2016) In vivo reprogramming for CNS repair: regenerating neurons from endogenous glial cells. Neuron 91 (4):728-738

33. Maraldi T, Bertoni L, Riccio M, Zavatti M, Carnevale G, Resca E, Guida M, Beretti F, La Sala GB, De Pol A (2014) Human amniotic fluid stem cells: neural differentiation in vitro and in vivo. Cell and tissue research $357(1): 1-13$

34. Markmee R, Aungsuchawan S, Narakornsak S, Tancharoen W, Bumrungkit K, Pangchaidee N, Pothacharoen P, Puaninta C (2017) Differentiation of mesenchymal stem cells from human amniotic fluid to cardiomyocyte-like cells. Molecular medicine reports 16 (5):6068-6076

35. Mercurio S, Serra L, Nicolis SK (2019) More than just Stem Cells: Functional Roles of the Transcription Factor Sox2 in Differentiated Glia and Neurons. International journal of molecular sciences 20 (18):4540

36. Nakagomi T, Taguchi A, Fujimori Y, Saino O, Nakano-Doi A, Kubo S, Gotoh A, Soma T, Yoshikawa H, Nishizaki T (2009) Isolation and characterization of neural stem/progenitor cells from post-stroke cerebral cortex in mice. European Journal of Neuroscience 29 (9):1842-1852

37. Nasrolahi A, Mahmoudi J, Karimipour M, Akbarzadeh A, Sadigh-Eteghad S, Salehi R, Farajdokht F, Farhoudi M (2019) Effect of cerebral dopamine neurotrophic factor on endogenous neural progenitor 
cell migration in a rat model of Parkinson's disease. EXCLI journal 18:139

38. Neuhuber B, Himes BT, Shumsky JS, Gallo G, Fischer I (2005) Axon growth and recovery of function supported by human bone marrow stromal cells in the injured spinal cord exhibit donor variations. Brain research 1035 (1):73-85

39. Niu W, Zang T, Zou Y, Fang S, Smith DK, Bachoo R, Zhang C-L (2013) In vivo reprogramming of astrocytes to neuroblasts in the adult brain. Nature cell biology 15 (10):1164-1175

40. Oh SK, Jeon SR (2016) Current concept of stem cell therapy for spinal cord injury: a review. Korean journal of neurotrauma $12(2): 40-46$

41. Ohori Y, Yamamoto S-i, Nagao M, Sugimori M, Yamamoto N, Nakamura K, Nakafuku M (2006) Growth factor treatment and genetic manipulation stimulate neurogenesis and oligodendrogenesis by endogenous neural progenitors in the injured adult spinal cord. Journal of Neuroscience 26 (46):11948-11960

42. Okada S, Nakamura M, Katoh H, Miyao T, Shimazaki T, Ishii K, Yamane J, Yoshimura A, Iwamoto Y, Toyama Y (2006) Conditional ablation of Stat3 or Socs3 discloses a dual role for reactive astrocytes after spinal cord injury. Nature medicine 12 (7):829-834

43. Osugi M, Katagiri W, Yoshimi R, Inukai T, Hibi H, Ueda M (2012) Conditioned media from mesenchymal stem cells enhanced bone regeneration in rat calvarial bone defects. Tissue engineering part A 18 (13-14):1479-1489

44. Pawitan JA (2014) Prospect of stem cell conditioned medium in regenerative medicine. BioMed research international 2014

45. Pischiutta F, Brunelli L, Romele P, Silini A, Sammali E, Paracchini L, Marchini S, Talamini L, Bigini P, Boncoraglio GB (2016) Protection of brain injury by amniotic mesenchymal stromal cell-secreted metabolites. Critical Care Medicine 44 (11):e1118-e1131

46. Rodriguez-Jimenez FJ, Alastrue A, Stojkovic M, Erceg S, Moreno-Manzano V (2016) Connexin 50 modulates Sox2 expression in spinal-cord-derived ependymal stem/progenitor cells. Cell and tissue research $365(2): 295-307$

47. Sabapathy V, Herbert FJ, Kumar S (2017) Therapeutic application of placental mesenchymal stem cells reprogrammed neurospheres in spinal cord injury of SCID. In: Adult Stem Cells. Springer, pp 91113

48. Saito S, Lin Y-C, Murayama Y, Hashimoto K, Yokoyama K (2012) Human Amnion-Derived cells as a reliable source of stem Cells. Current molecular medicine 12 (10):1340-1349

49. Salehi-Pourmehr H, Rahbarghazi R, Mahmoudi J, Roshangar L, Chapple CR, Hajebrahimi S, Abolhasanpour N, Azghani M-R (2019) Intra-bladder wall transplantation of bone marrow mesenchymal stem cells improved urinary bladder dysfunction following spinal cord injury. Life sciences 221:20-28

50. Shahrezaie M, Mansour RN, Nazari B, Hassannia H, Hosseini F, Mahboudi H, Eftekhary M, Kehtari M, Veshkini A, Vasmehjani AA (2017) Improved stem cell therapy of spinal cord injury using GDNFoverexpressed bone marrow stem cells in a rat model. Biologicals 50:73-80 
51. Shi Y, Hu G, Su J, Li W, Chen Q, Shou P, Xu C, Chen X, Huang Y, Zhu Z (2010) Mesenchymal stem cells: a new strategy for immunosuppression and tissue repair. Cell research 20 (5):510-518

52. Sofroniew MV (2009) Molecular dissection of reactive astrogliosis and glial scar formation. Trends in neurosciences 32 (12):638-647

53. Sofroniew MV, Vinters HV (2010) Astrocytes: biology and pathology. Acta neuropathologica 119 (1):7-35

54. Springer JE, Visavadiya NP, Sullivan PG, Hall ED (2018) Post-injury treatment with NIM811 promotes recovery of function in adult female rats after spinal cord contusion: a dose-response study. Journal of neurotrauma 35 (3):492-499

55. Su Z, Niu W, Liu M-L, Zou Y, Zhang C-L (2014a) In vivo conversion of astrocytes to neurons in the injured adult spinal cord. Nature communications 5 (1):1-15

56. Su Z, Niu W, Liu M-L, Zou Y, Zhang C-L (2014b) In vivo conversion of astrocytes to neurons in the injured adult spinal cord. Nature communications 5:3338

57. Sun X, Tan Z, Huang X, Cheng X, Yuan Y, Qin S, Wang D, Hu X, Gu Y, Qian W-J (2019) Direct neuronal reprogramming of olfactory ensheathing cells for CNS repair. Cell death \& disease 10 (9):1-17

58. Teixeira F, Pires A, Fraga J, Carvalho M, Silva N, Panchalingam K, Jung S, Sousa N, Behie L, Salgado A (2014) Secretome of mesenchymal stem cells as a new tool for central nervous system regenerative medicine.

59. Torper O, Pfisterer U, Wolf DA, Pereira M, Lau S, Jakobsson J, Björklund A, Grealish S, Parmar M (2013) Generation of induced neurons via direct conversion in vivo. Proceedings of the National Academy of Sciences 110 (17):7038-7043

60. Tsai M-S, Hwang S-M, Tsai Y-L, Cheng F-C, Lee J-L, Chang Y-J (2006) Clonal amniotic fluid-derived stem cells express characteristics of both mesenchymal and neural stem cells. Biology of reproduction 74 (3):545-551

61. Uccelli A, Benvenuto F, Laroni A, Giunti D (2011) Neuroprotective features of mesenchymal stem cells. Best practice \& research Clinical haematology 24 (1):59-64

62. van Koppen A, Joles JA, van Balkom BW, Lim SK, de Kleijn D, Giles RH, Verhaar MC (2012) Human embryonic mesenchymal stem cell-derived conditioned medium rescues kidney function in rats with established chronic kidney disease. PloS one 7 (6):e38746

63. Wang L-L, Su Z, Tai W, Zou Y, Xu X-M, Zhang C-L (2016) The p53 pathway controls SOX2-mediated reprogramming in the adult mouse spinal cord. Cell reports 17 (3):891-903

64. Yiu G, He Z (2006) Glial inhibition of CNS axon regeneration. Nature Reviews Neuroscience 7 (8):617627

65. Zhang S, Cui W (2014) Sox2, a key factor in the regulation of pluripotency and neural differentiation. World journal of stem cells 6 (3):305

\section{Supplementary Files}


This is a list of supplementary files associated with this preprint. Click to download.

- SOX2.tif 